\title{
Zigzag chain model and its spectrum
}

\author{
A. S. Melikhova \\ ITMO University, Kronverkskiy, 49, St. Petersburg, 197101, Russia \\ alina.s.melikhova@gmail.com
}

PACS 02.30.Tb

DOI 10.17586/2220-8054-2017-8-2-188-193

This work describes the development of a model using a zigzag chain of weakly-coupled ball resonators with Neumann boundary conditions. The chain is assumed to be constructed of identical resonators connected through point-like apertures. The connecting points are described by their delta-coupling with a constant intensity. The model is based on the theory of self-adjoint extensions of symmetrical operators. Due to effectively one-dimensional joints, the $3 \mathrm{D}$ problem can be solved with assistance from the transfer matrix approach. This allows us to study the spectrum of the physical system. In particular, it is proven that the discrete spectrum of direct zigzag chain is empty while bending deformation leads to the appearance of non-empty discrete spectrum. In addition, the continuous spectrum has band structure. With the help of asymptotic study, we obtain the dependence of the spectrum structure on the geometrical and physical parameters of the system: zigzag angle, bend angle and coupling intensity.

Keywords: bending deformation, extension theory, transfer-matrix approach, discrete spectrum.

Received: 18 January 2017

Revised: 18 February 2017

\section{Introduction}

Various chain structures have been widely discussed recently. These structures can be constructed of elements of different dimensions. Regarding one-dimensional elements, spectral properties of an infinite chain-like quantum graph, which consists of identical rings, were studied in [1,2]. In [3] authors deal with a similar chain made up of hexagons. Two-dimensional resonators are in the focus of the papers [4-6], which studies spectral problems of direct and zigzag-like chains of disks, and bent chain of nanospheres respectively. Finally, chains consisting of three-dimensional resonators are presented in [7-9].

Different chains structures with resonators as cells are used in optical systems (see, for example, so called SCISSOR device [10], optical waveguide system [11], optical delay line [12]), in nanoelectronics (see, for instance, nano peapod and similar systems [13-17]) and in molecular biology (for example, for creation of biosensors [18]).

The spectrum of stationary Schrödinger equation in the zigzag-like chain system will be examined in the present paper. The Hamiltonian for this model is constructed in the framework of extension's theory (see, for example, [19] and the extensive bibliography of [20]). We assume that resonators in our model are weakly coupled, i. e. we employ the approach known as model of zero-width slit [21-24]. We consider Neumann boundary conditions at resonator's border and $\delta$-coupling condition at its contact points. In this paper, we analyze the dependence of spectral characteristics on the geometrical and physical parameters of the system.

\section{Model and geometry}

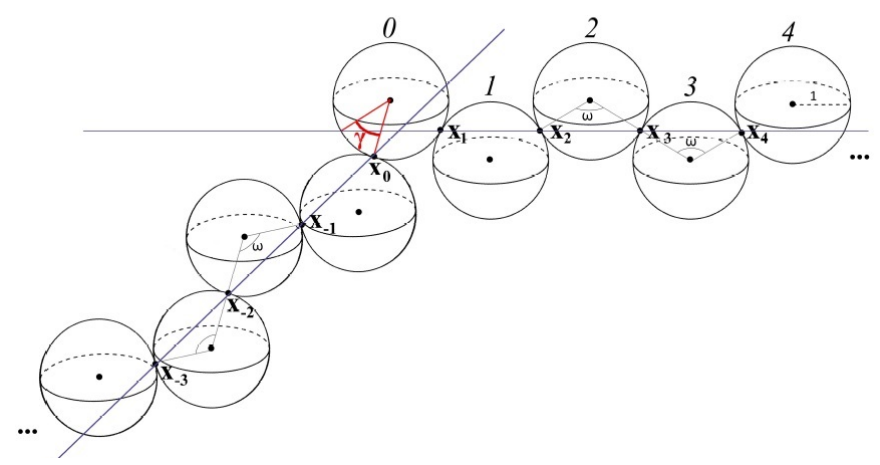

FIG. 1. Geometry of the system 
The three-dimensional zigzag chain of weakly coupled ball resonators with a kink is considered (see Fig. 1). From geometrical point of view this system has the following properties:

- it is made up of infinite quantity of identical ball resonators (it is convenient to enumerate them: $j \in$ $\mathbb{Z}_{-} \cup\{0\} \cup \mathbb{Z}_{+}$). Without loss of generality, we assume that all balls are of unit radius;

- any two neighboring resonators $(j-1$ and $j)$ have only one common point $\left(\mathbf{x}_{j}\right)$. Before applying "bending" deformation all such contact points lie on the same straight line;

- $\omega$ is the zigzag angle: $\omega \in(\pi / 3 ; \pi]$;

- any two connected resonators can be taken as a basic cell;

- "bending" deformation (kink) occurs at a single point $\left(\mathbf{x}_{0}\right)$ shifted by an angle $\gamma: \gamma \in[0 ; \omega-\pi / 3)$.

When geometry of the system is comprehended, the Hamiltonian can be described. We employ the theory of self-adjoint extensions of symmetric operator to present states of non-relativistic spinless particles that are placed into the chain in the absence of external fields. For a better understanding of the restriction-extension scheme which is used for model construction, let one examine more scrupulously the simplest chain - two connected resonators. And then one just needs to extend this scheme for the infinite case.

Consider two coupled resonators $\left(\Omega_{0}, \Omega_{1}\right)$ with one common point $\mathbf{x}_{1}$ at the border. Let $-\Delta$ be the orthogonal sum of the Laplace operators with Neumann boundary conditions in $L_{2}\left(\Omega_{0}\right) \oplus L_{2}\left(\Omega_{1}\right)$. Restrict this operator to the set of smooth functions vanishing at contact point $\mathbf{x}_{1}$. This procedure gives us symmetric operator $-\Delta_{0}$ with deficiency indices $(2,2)$, however, it is a non-self-adjoint operator. Its self-adjoint extension gives us the model of resonators coupled through point-like aperture. It is clear that the self-adjoint extension is a restriction of the adjoint operator. Therefore, one should first describe the adjoint operator. This can be done in several ways. For example, this can be done using the so-called boundary triplet approach (see, for example, [25, 26]) or the Von Neumann's formulas can be used (see, for instance, [27, 28]). In our case, the domain of the adjoint operator in our model (due to the positivity of the operator and, correspondingly, regularity of negative points at the real axis) can be written in such manner:

$$
D\left(-\Delta_{0}^{*}\right)=D\left(-\Delta_{0}^{F}\right)+N_{\lambda_{0}},
$$

where $D\left(-\Delta_{0}^{F}\right)$ is the Friedrichs extension of our restricted operator and $N_{\lambda_{0}}$ is a deficiency subspace that refers to negative real regular point $\lambda_{0}$ (see, for example, [29]). Correspondingly, elements $f \in D\left(-\Delta_{0}^{*}\right)$ have such form:

$$
f=\left(\begin{array}{c}
f_{0} \\
f_{1}
\end{array}\right)=\left(\begin{array}{l}
f_{0}^{0}+a_{0}^{-} G_{0}\left(\mathbf{x}, \mathbf{x}_{1}, \lambda_{0}\right)+b_{0}^{-} \\
f_{1}^{0}+a_{1}^{+} G_{1}\left(\mathbf{x}, \mathbf{x}_{1}, \lambda_{0}\right)+b_{1}^{+}
\end{array}\right),
$$

where $f_{i}^{0} \in D\left(-\Delta_{0}\right), i=0,1$ and $a_{0}^{-}, a_{1}^{+}, b_{0}^{-}, b_{1}^{+}$are some coefficients (by signs " + " and "-" nearby $i$-th coefficient we distinguish ones that refer to $i$-th resonator but to different contact points). By establishing the cross-linking between these coefficients the self-adjoint operator can be obtained.

These algorithm can be quite easily extended to the case of an infinite chain. In this case the initial (before including the interactions between resonators) operator is $-\Delta:-\Delta=\bigoplus_{j \in \mathbf{Z}}\left(-\Delta_{j}\right)$ in $\bigoplus_{j \in \mathbf{Z}} L_{2}\left(-\Delta_{j}\right)$. Moreover, this operator should be restricted on the set of all smooth functions from $D(-\Delta)$ that vanish at contact points $\mathbf{x}_{j}$. Such restriction is a symmetric operator with deficiency indices $(\infty, \infty)$ and Green's functions $G\left(\mathbf{x}, \mathbf{x}_{j}, \lambda_{0}\right)$ as deficiency elements.

An adjoint operator for an infinite chain is constructed similarly to the simple case of two resonators. Finally, the self-adjointness condition is satisfied with:

$$
\left\{\begin{array}{l}
a_{j}^{+}=-a_{j-1}^{-}, \\
b_{j}^{+}=b_{j-1}^{-},
\end{array}\right.
$$

where coefficients $b_{j}^{ \pm}$are defined by coefficients $a_{j}^{ \pm}$:

$$
\begin{gathered}
b_{j}^{+}=a_{j}^{+} \lim _{\mathbf{x} \rightarrow \mathbf{x}_{j}}\left(G\left(\mathbf{x}, \mathbf{x}_{j}, \lambda\right)-G\left(\mathbf{x}, \mathbf{x}_{j}, \lambda_{0}\right)\right)+a_{j}^{-} G\left(\mathbf{x}_{j+1}, \mathbf{x}_{j}, \lambda\right), \\
b_{j-1}^{-}=a_{j-1}^{+} G\left(\mathbf{x}_{j-1}, \mathbf{x}_{j}, \lambda\right)+a_{j-1}^{-} \lim _{\mathbf{x} \rightarrow \mathbf{x}_{j}}\left(G\left(\mathbf{x}, \mathbf{x}_{j}, \lambda\right)-G\left(\mathbf{x}, \mathbf{x}_{j}, \lambda_{0}\right)\right) .
\end{gathered}
$$

Moreover, when our model is supplemented by $\delta$-coupling conditions at contact points $\mathbf{x}_{j}$ (with coupling constant $\alpha \in \mathbb{R}$ ), system of equations (1) transforms to:

$$
\left\{\begin{aligned}
a_{j}^{+} & =-a_{j-1}^{-}, \\
b_{j}^{+}-b_{j-1}^{-} & =-\alpha a_{j-1}^{-} .
\end{aligned}\right.
$$




\section{Spectral analysis}

The system being under investigation is periodic in part, and the transfer-matrix approach is a good tool for spectral analysis (see, e.g., [30]); in fact, that the asymptotic behavior of the $\left\|\left(\begin{array}{ll}a_{j}^{+} & a_{j}^{-}\end{array}\right)^{T}\right\|$ is determined by the spectral properties of the transfer-matrix.

First, we consider a simple zigzag chain without kinks. By serial employment of the formulas (2)-(4), one can easily obtain the matrix relation between coefficients $a_{j-1}^{ \pm}$and $a_{j+1}^{ \pm}$as follows:

$$
\begin{aligned}
\left(\begin{array}{l}
a_{j+1}^{+} \\
a_{j+1}^{-}
\end{array}\right) & =\mathbf{M}\left(\begin{array}{c}
a_{j-1}^{+} \\
a_{j-1}^{-}
\end{array}\right), \\
\mathbf{M}=\mathbf{M}_{j-1 \rightarrow j} \mathbf{M}_{j \rightarrow j+1} & =\left(\begin{array}{cc}
-1 & -\frac{2 g-\alpha}{G_{\omega}} \\
\frac{2 g-\alpha}{G_{\omega}} & \left(\frac{2 g-\alpha}{G_{\omega}}\right)^{2}-1
\end{array}\right),
\end{aligned}
$$

where $g=\lim _{\mathbf{x} \rightarrow \mathbf{x}_{j}}\left(G\left(\mathbf{x}, \mathbf{x}_{j}, \lambda\right)-G\left(\mathbf{x}, \mathbf{x}_{j}, \lambda_{0}\right)\right), G_{\omega}=G\left(\mathbf{x}_{j}, \mathbf{x}_{j+1}, \lambda\right)(j \neq 0)$ and $\mathbf{M}$ is a transfer-matrix that permits one to find proper conditions for spectral analysis. Namely, the value $\lambda$ belongs to the continuous spectrum if the absolute value of the corresponding eigenvalue $\mu$ of matrix $\mathbf{M}$ is equal to one. Whereas for belonging of the value $\lambda$ to the discrete spectrum it is necessary for the corresponding eigenvalue $\mu$ of matrix $\mathbf{M}$ in absolute value to be less than one.

From (5), one can find the explicit form of the transfer-matrix's eigenvalues and eigenvectors:

$$
\begin{gathered}
\mu_{ \pm}=\frac{\left((2 g-\alpha) / G_{\omega}\right)^{2}}{2}-1 \pm \sqrt{\left(\frac{\left((2 g-\alpha) / G_{\omega}\right)^{2}}{2}-1\right)^{2}-1} \\
\nu_{ \pm}=\left(\begin{array}{c}
\left.-\frac{2 g-\alpha}{2 G_{\omega}} \pm \sqrt{\left(\frac{2 g-\alpha}{2 G_{\omega}}\right)^{2}-1}\right) . \\
1
\end{array}\right)
\end{gathered}
$$

Thus, keeping in mind the above-mentioned spectral properties of the transfer-matrix and (6), one can obtain the inequality that describes the band structure of the continuous spectrum of the model Hamiltonian:

$$
\left|\frac{2 g-\alpha}{G_{\omega}}\right| \leq 2
$$

It is clear that the presence of one kink in the chain (introduced as a shift of one contact point) does not affect the band structure. However, such bending deformation potentiates the appearance of bound states in the gaps.

Let us now examine the bending area more accurately. In order to find a decreasing at infinity solution, one should first merge two semi-infinite chains via a new transfer-matrix $\mathbf{M}_{\gamma}$ :

$$
\mathbf{M}_{\gamma}=\mathbf{M}_{-1 \rightarrow 0} \mathbf{M}_{0 \rightarrow-1}=\left(\begin{array}{cc}
-\frac{G_{\gamma}}{G_{\omega}} & -\frac{2 g-\alpha}{G_{\omega}} \\
-\frac{2 g-\alpha}{G_{\omega}} & \frac{(2 g-\alpha)^{2}-G_{\omega}^{2}}{G_{\gamma} G_{\omega}}
\end{array}\right),
$$

where $G_{\gamma}=G\left(\mathbf{x}_{0}, \mathbf{x}_{1}, \lambda\right)$. And then, one should satisfy the linear dependence condition:

$$
\left|\begin{array}{ll}
a_{1}^{+} & a_{-1}^{+} \\
a_{1}^{-} & a_{-1}^{-}
\end{array}\right|=0
$$

where $\left(\begin{array}{ll}a_{-1}^{+} & a_{-1}^{-}\end{array}\right)^{T}=\zeta \nu_{ \pm}$. By substituting expressions (5), (7) and (9) into condition (10), one can obtain the spectral equation in the most simple form as follows:

$$
\frac{G_{\gamma}}{G_{\omega}}-\frac{G_{\omega}}{G_{\gamma}}+\left(\frac{2 g-\alpha}{G_{\omega}}\right)^{2}\left(\frac{G_{\omega}}{G_{\gamma}}-1\right)=0 .
$$

Finally, one should take into account that the solution $\lambda^{*}$ of (11) must satisfy the corresponding condition on $\mu$ :

$$
\left.\left|\frac{2 g-\alpha}{G_{\omega}}\right|\right|_{\lambda^{*}}>2 \text {. }
$$

Thus, the solutions of problem (11)-(12) draws up the discrete spectrum of the model Hamiltonian. 


\section{Results and discussions}

Gathering all facts mentioned above, the Theorem representing spectrum of the model Hamiltonian is stated: Theorem. Let the bend angle $\gamma$ of the chain belong to $[0 ; \omega-\pi / 3)$. The continuous spectrum has band structure and is given by Equation (8). There are eigenvalues of infinite multiplicity which are given by the eigenvalues of the Neumann Laplacian for the ball corresponding to the eigenfunctions vanishing at the both opposite points of the ball $\left(\mathbf{x}_{j}\right.$ and $\left.\mathbf{x}_{j-1}\right)$. If there is no bending deformation $(\gamma=0)$, the discrete spectrum is empty. There exist values of the model parameters $\alpha, \gamma, \lambda_{0}$ and $\omega$ such that the model Hamiltonian has eigenvalues in the gaps in the case with $\gamma \neq 0$ and these eigenvalues are given by (11)-(12).

Figures 2-5 depict the results of numerical modelling of the spectral problem. From these graphs, one can see how changing the parameters influence its spectral characteristics (gap widths, band's splitting (Fig. 2-4) and eigenvalue's appearance (Fig. 5)).

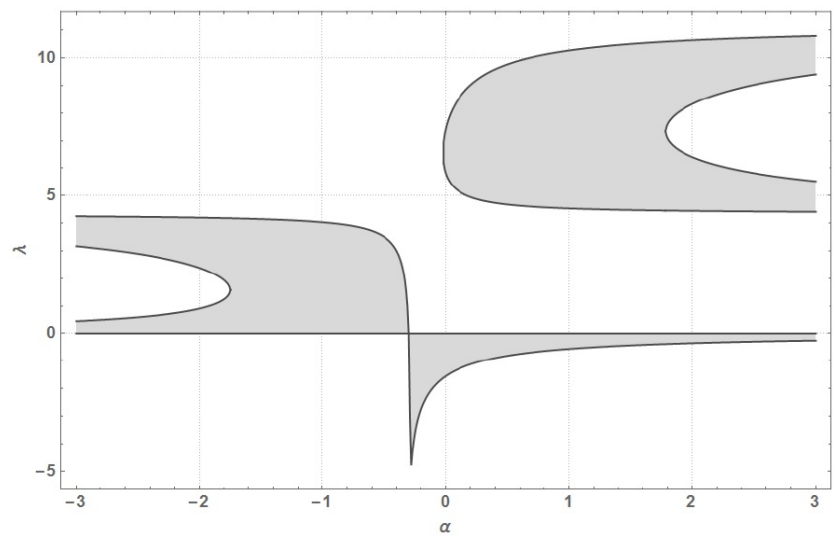

FIG. 2. Band structure of continuous spectrum depending on $\alpha$ (with $\omega=5 \pi / 6, \gamma=0$ )

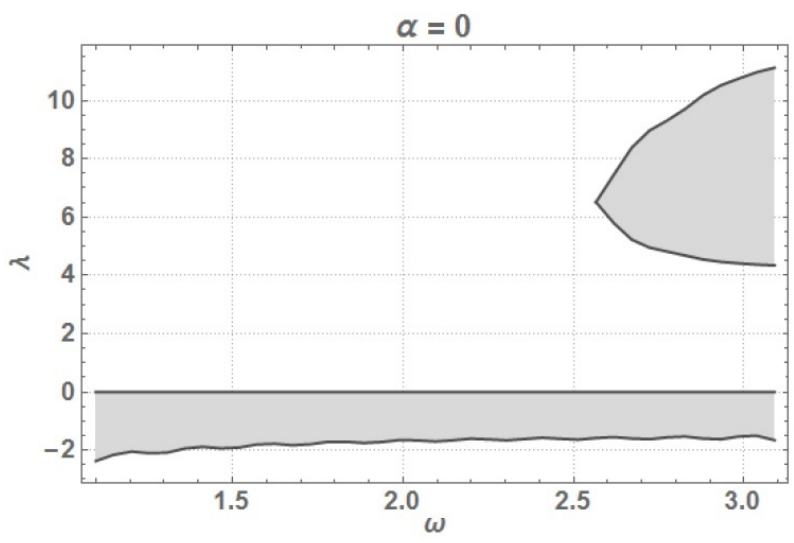

FIG. 3. Band structure of continuous spectrum depending on $\omega$ during "free particle motion" $(\alpha=0, \gamma=0)$

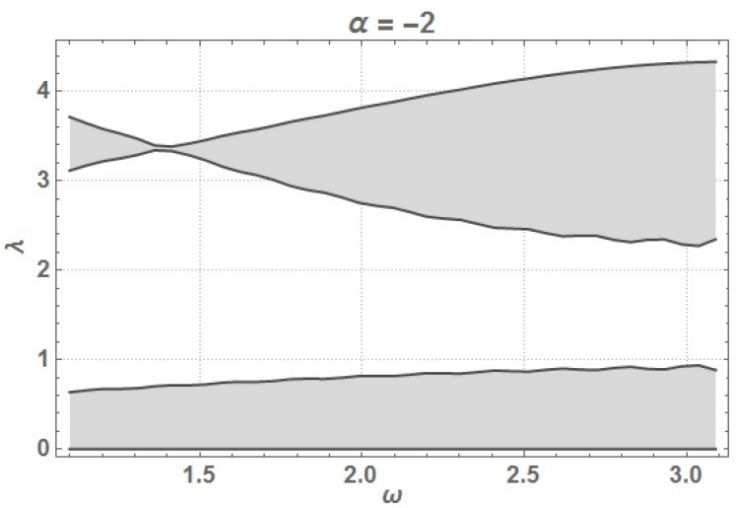

FIG. 4. Band structure of continuous spectrum depending on $\omega$ in the presence of $\delta$-potentials at contact points $\mathbf{x}_{j}(\gamma=0)$

Furthermore, analytical expressions for the lowest energy band can be obtained. Specifically, the asymptotic expressions for borders of the first energy band can be gained from (8) when $\lambda$ tends to zero:

$$
\begin{gathered}
\lambda_{1}=\frac{3}{2 \pi}\left(\frac{3}{4 \pi \lambda_{0}}-\frac{\alpha}{2}+\sum_{k=1, l=0}^{\infty} \frac{N_{l, k}}{x_{l, k}^{2}}\left(P_{l}(\cos \omega)-\frac{\lambda_{0}}{x_{l, k}^{2}-\lambda_{0}}\right)\right)^{-1}+\left(\lambda_{1}^{2}\right), \\
\lambda_{2}=\left[\frac{\alpha}{2}-\frac{3}{4 \pi \lambda_{0}}+\sum_{k=1, l=0}^{\infty}\left(-\frac{N_{l, k}}{x_{l, k}^{2}}\left(\frac{\lambda_{0}}{x_{l, k}^{2}-\lambda_{0}}+P_{l}(\cos \omega)\right)\right)\right]\left[\sum_{k=1, l=0}^{\infty} \frac{N_{l, k}\left(1-P_{l}(\cos \omega)\right)}{x_{l, k}^{4}}\right]^{-1}+O\left(\lambda_{2}^{2}\right),
\end{gathered}
$$



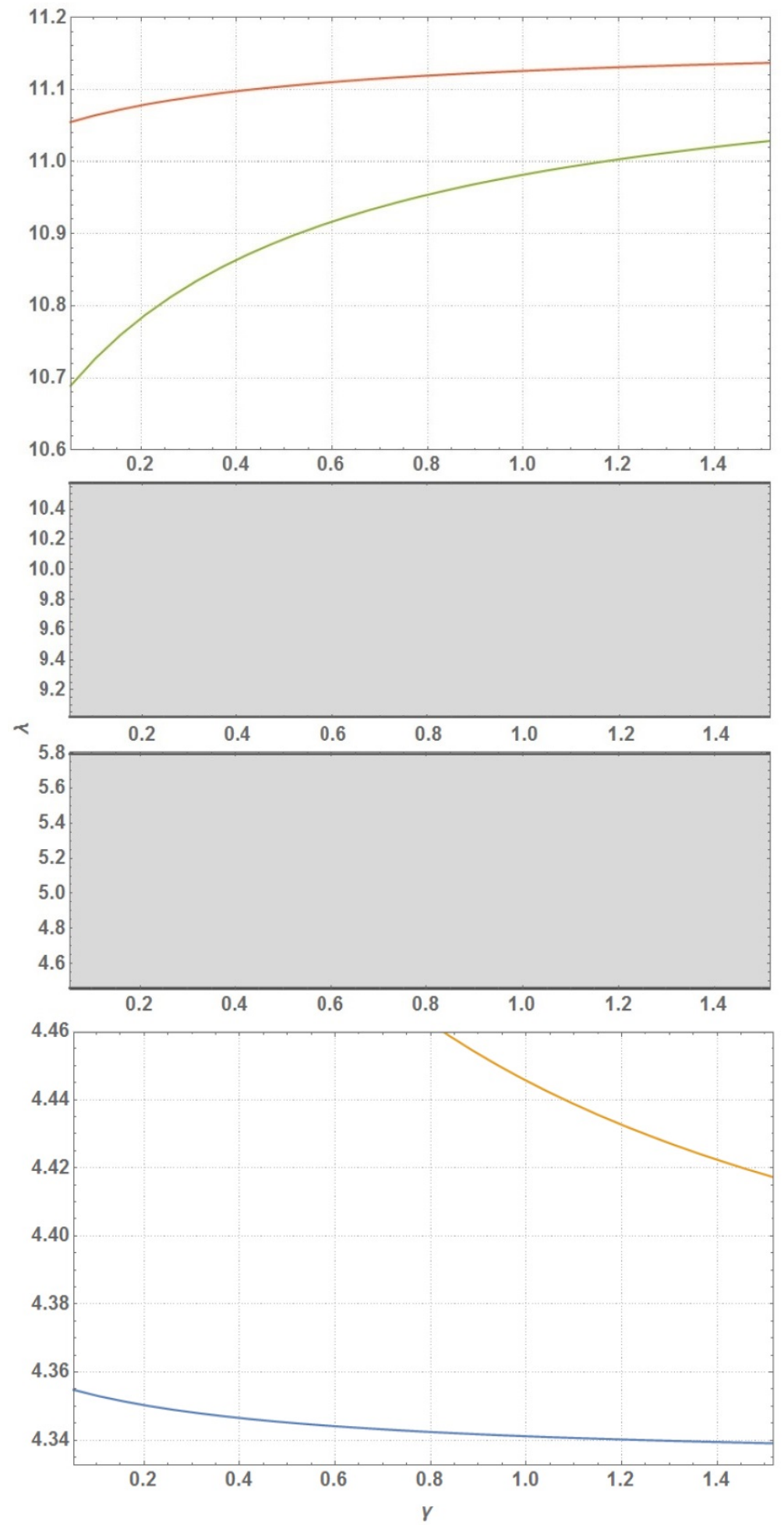

FIG. 5. Spectral structure depending on bending angle $\gamma$ (with $\alpha=2$ and $\omega=5 / 6$ ): grey bands - the two neighboring bands of continuous spectrum (do not depend on $\gamma$ ), curves values that belong to the discrete spectrum 
where $x_{l, k}$ is a $k$-th root of the equation: $j_{l}^{\prime}(x)=0$ and $N_{l, k}$ is a normalization factor.

Thus, this model allows us to obtain both analytical and numerical results for a non-trivial three-dimensional system. Finally, the described model allows us to exert an influence on the spectrum of the zigzag-like chain of resonators. Variation of the model parameters is a tool which can be utlized to control and modify the spectrum structure.

\section{Acknowledgements}

This work was partially financially supported by the Government of the Russian Federation (grant 074-U01), by grant MK-5161.2016.1 of the President of the Russian Federation, by grant 16-11-10330 of Russian Science Foundation.

\section{References}

[1] Duclos P., Exner P., Turek O. On the spectrum of a bent chain graph. J. Phys. A: Math. Theor., 2008, 41, P. 415206.

[2] Popov I. Yu., Smirnov P. I. Spectral problem for branching chain quantum graph. Phys. Lett. A, 2013, 377(6), P. 439-442.

[3] Popov I. Yu., Skorynina A. N., Blinova I. V. On the existence of point spectrum for branching strips quantum graph. J. Math. Phys., 2014, 55, P. 033504.

[4] Grishanov E. N., Eremin D. A., Ivanov D. A., Popov I. Yu. Periodic chain of disks in a magnetic field: bulk states and edge states. Nanosystems: Phys. Chem. Math., 2015, 6(5), P. 637-643.

[5] Blinova I. V., Melikhova A.S., Popov I. Yu. Periodic chain of resonators: gap control and the system geometry. Journal of Physics: Conference Series, 2016, 735, P. 012062.

[6] Eremin D. A., Ivanov D. A., Popov I. Yu. Electron energy spectrum for a bent chain of nanospheres. Eur. Phys. J. B, $2014,87(8)$, P. 181.

[7] Melikhova A. S., Popov I. Yu. Spectral problem for solvable model of bent nano peapod. Applicable Analysis, 2017, 96(2), P. $215-224$.

[8] Melikhova A.S. Estimates for numbers of negative eigenvalues of Laplacian for Y-type weakly coupled chain of ball resonators. Mathematical Results in Quantum Mechanics. Proceedings of the QMath12 Conference, 2014, P. 325-330.

[9] Melikhova A. S., Popov I. Yu. Bent and branched chains of nanoresonators. Journal of Physics: Conference Series, 2014,541, P. 012061.

[10] Heebner J. E., Boyd R. W., and Park Q.-H. SCISSOR solitons and other novel propagation effects in microresonator-modified waveguides. J. Opt. Soc. Am. B, 2002, 19, P. 722-731.

[11] Popov I. Yu., Trifanov A. I. and Trifanova E. S. Coupled dielectric waveguides with photonic crystal properties. Comput. Math. Math. Phys, 2010, 50(11), P. 1830-1836.

[12] Lenz G., Eggleton B. J., Madsen C. K., and Slusher L. E. Optical Delay Lines Based on Optical Filters. IEEE J. Quantum Electron, 2001, 37(4), P. 525-532.

[13] Wang Y., Zhang H.J., Lu L., Stubbs L.P., Wong C.C., Lin J. Designed Functional Systems from Peapod-like Co@Carbon to $\mathrm{Co}_{3} \mathrm{O}_{4} @$ Carbon Nanocomposites. ACS Nano, 2010, 4(8), P. 4753-4761.

[14] Yang Y., Li L., Li W. Plasmon Absorption of Au-in-CoAl2O4 Linear Nanopeapod Chains. J. Phys. Chem. C, 2013, 117(27), P. 1414214148.

[15] Enyashin A. N., Ivanovskii A. L. Nanotubular composites: modeling of capillary filling of nanotubes of disulfide of molybdenum by molecules of TiCl4. Nanosystems: Phys. Chem. Math, 2010, 1(1), P. 63-71.

[16] Krivanek O. L., Dellby N.,.Murfitt M.F, Chisholm M.F., Pennycook T. J., Suenaga K. and Nicolosi V. Gentle STEM: ADF imaging and EELS at low primary energies. Ultramicroscopy, 2010, 110(8), P. 935-945.

[17] Abou-Hamad E., Kim Yo., Talyzin A. V., Goze-Bac Ch., Luzzi D. E., Rubio A., Wagberg T. Hydrogenation of C60 in Peapods: Physical Chemistry in Nano Vessels. J. Phys. Chem. C, 2009, 113, P. 8583-8587.

[18] Boyd R. W. and Heebner J. E. Sensitive disk-resonator photonic biosensor. Appl. Opt., 2001 , 40(31), P. $5742-5747$.

[19] Albeverio S., Gestesy F., Hoegh-Krohn R., et al. Solvable models in quantum mechanics. Springer-Verlag, Berlin, 1988.

[20] Popov I. Y., Kurasov P. A., Naboko S. N., Kiselev A. A., Ryzhkov A. E., Yafyasov A. M., Miroshnichenko G. P., Kareshina E. Yu., Kruglov V. I., Pankratova T. F., Popov A. I. A distinguished mathematical physicist Boris S. Pavlov. Nanosystems: Phys., Chem., Math., 2016, 7(5), P. 782-788.

[21] Popov I. Yu. The resonator with narrow slit and the model based on the operator extensions theory. J. Math. Phys., 1992, 33(11), P. 3794-3801.

[22] Popov I. Yu. 2012 Operator extensions theory model for electromagnetic field-electron interaction. J. Math. Phys., 2012, 53(6), P. 063505.

[23] Popov I. Yu. Extensions theory and localization of resonances for domains of trap type. Mat. Sbornik, 1990, 181(10), P. 1366-1390.

[24] Popov I. Yu. Model of point-like window for electromagnetic Helmholtz resonator. Zeitschrift Anal. Anwend., 2013, 32, P. 155-162.

[25] Behrndt J., Langer M., Lotoreichik V. Boundary triples for Schrdinger operators with singular interactions on hypersurfaces. Nanosystems: Phys., Chem., Math., 2016, 7(2), P. 290-302.

[26] Boitsev A. A., Neidhardt H., Popov I. Yu. Dirac operator coupled to bosons. Nanosystems: Phys., Chem., Math., 2016, 7(2), P. 332-339.

[27] Geyler V. A., Pavlov B. S., Popov I. Yu. Spectral properties of a charged particle in antidot array: A limiting case of quantum billiard. J. Math. Phys., 1996, 37(10), P. 5171-5194.

[28] Berndt J., Malamud M., Neidhardt H. Scattering matrices and Weyl functions. Proc. London Math. Soc., 2008, 97(3), P. 568-598.

[29] Birman M. Sh., Solomjak M.Z. Spectral Theory of Self-Adjoint Operators in Hilbert Space. D. Reidel Publishing Company, Dordrecht, 1987, $301 \mathrm{pp}$.

[30] Anikevich A.S. Negative eigenvalues of the Y-type chain of weakly coupled ball resonators. Nanosystems: Phys. Chem. Math., 2013, 4(4), P. 545-549. 